\title{
Degradation Detection for Internal Gear Pumps using Pressure Reduction Time Maps
}

\author{
Kurt Pichler ${ }^{1}$, Rainer Haas ${ }^{2}$, Veronika Putz ${ }^{3}$, and Christian Kastl ${ }^{4}$ \\ 1,3, 4 Linz Center of Mechatronics GmbH, Area Sensors \& Communication, 4040 Linz, Austria \\ kurt.pichler@lcm.at \\ veronika.putz@lcm.at \\ christian.kastl@lcm.at \\ ${ }^{2}$ Linz Center of Mechatronics GmbH, Area Drives, 4040 Linz, Austria \\ rainer.haas@lcm.at
}

\begin{abstract}
In this paper, a novel approach for detecting degradation in internal gear pumps is proposed. In a data-driven approach, pressure reduction time maps (PRTMs) are identified as a useful indicator for degradation detection. A PRTM measures the time for reducing the internal pump pressure from certain levels to any lower level when the pump engine is stopped and the valves are closed. The PRTM can thus be interpreted as an internal leakage indicator of the pump. For simplified evaluation, PRTMs are compressed to a single scalar indicator by computing their volume (PRTMV). When the internal leakage increases due to wear, the pressure in the pump decreases faster (implying a decreased PRTMV). The proposed approach has been developed and tested with data of real internal gear pumps with different operating times. The PRTMV shows a close relation to the operating time of the pump. Moreover, we compare PRTMV to the commonly used and well known approach of observing pressure holding speed (PHS). Especially for medium degradation, PRTMV shows better sensitivity than PHS.
\end{abstract}

\section{INTRODUCTION}

Internal gear pumps are nowadays widely used, for instance to pump all varieties of fuel oil and lube oil, resins, polymers, alcohols, solvents, asphalt, bitumen, tar, polyurethane foam, food products such as chocolate, paint, ink, soaps, surfactants or glycol. The common trend in industry goes towards higher safety and economical demands, this is also true for pumps. Customers expect to reduce unplanned downtime as well as extended maintenance intervals. Using condition monitoring and predictive maintenance, the maintenance in-

\footnotetext{
Kurt Pichler et al. This is an open-access article distributed under the terms of the Creative Commons Attribution 3.0 United States License, which permits unrestricted use, distribution, and reproduction in any medium, provided the original author and source are credited.
}

tervals of a component can be optimized compared to maintaining in fixed time intervals without considering the actual health state.

Moreover, automated technical systems to detect component failure help reducing manual inspections and have therefore the capability to save a significant amount of personnel cost. Additionally, the development of a condition monitoring system oftentimes improves the understanding of a process. This can be used for evaluation and recommendations regarding machine operation.

Wear and damage detection for pumps is a well researched field in engineering, many publications on this topic can be found. A broad overview of condition monitoring for pumps is given in (Beebe, 2005). Typical symptom parameters for condition diagnosis in frequency domain are extracted from vibration measurements in (Wang \& Chen, 2007). In (Mendel, Wandekokem, Rauber, \& Varejão, 2007), characteristic frequencies for fault states are identified using feature selection in frequency spectra. Visual inspection of head flow diagrams and vibration charts in (Albraik, Althobiani, Gu, \& Ball, 2012) showed a different behavior of new and damaged pumps. In (Zouari, Sieg-Zieba, \& Sidahmed, 2004), five fault states are discriminated by training neural networks with features extracted from the frequency spectrum of vibration measurements. Voltage and current of a pump motor are modeled with an Auto Regressive eXogenous (ARX) model, and subsequently fault indicators are extracted from the measurement data and from the models respectively in (Harihara \& Parlos, 2012). In (Byington, Watson, Edwards, \& Dunkin, 2003), features from pressure, temperature and flow rate measurements are extracted and classified with neural networks. In (Ahonen, 2011), cavitation is detected via deviations of rotational speed and torque, while flow recirculation is detected via spectral analysis of the torque. The change of head flow 
diagram in the case of pump damages is discussed in (Beebe, 2008). In (Cotogno, Cocconcelli, \& Rubini, 2013), air bubbles in internal gear pumps are detected by extracting features from vibration data in time and frequency domain and thresholding the features. The typical fault types of internal gear pumps and their impact on the operating pressure are discussed in (Pietkiewicz, 2009). In (Singh, Tjahjowidodo, Eriksson, \& Rajan, 2018), the torque of a reciprocating pump is analyzed to perform condition monitoring. Frequency band energy information of wavelet packet decomposition are extracted as features and compared to test samples achieved by Q statistics to detect faults in hydraulic pumps in (Siyuan, Linlin, \& Wanlu, 2018). Indicators for seal damage and cavitation are extracted from the frequency spectra of vibration measurements in (Sun, Yuan, \& Luo, 2018). In (Dutta et al., 2018), cavitation is detected using support vector machines for classifying pressure and speed measurements. In (Sanchez, Carvajal, Poalacin, \& Salazar, 2018), an alteration in the vibration range at the alabe pitch frequency is reported in the case of cavitation.

Many of the recently proposed concepts could not be used in our experiments due to limited measurement capacities like limited access to machine parameters (for instance, the actual driving current or torque is not accessible), no direct access to the flowing liquid, and rigid cost constraints. Therefore, a novel data-driven approach is investigated in this study. Data of internal gear pumps with known operating time (assumed to be correlated to degradation) are acquired and analyzed to develop a degradation indicator. Faults are detected using only a limited number of predefined measurement channels, including internal pump pressure and rotational speed. Measurement data from pumps with different operating times are compared in a data-driven approach to define an appropriate quantifier. Analyzing the data yields in a single scalar value called pressure reduction time map volume (PRTMV) that is extracted from measured data and subsequently observed. A decreasing PRTMV value corresponds to increasing operating time of the pump.

Compared to other approaches like pressure holding speed (PHS), the proposed approach takes the dynamic behavior of the oil pressure into account, as mentioned in Section 3. Therefore it is more sensitive in the medium wear range of the pump than the approaches that only monitor the steady state pressure. A drawback of the PRTMV approach is of course that a certain test pattern is needed, see also Section 3 . The pump has to be stopped at a certain constant pressure level with closed valves, and the pressure drop has to be measured. However, this is quite similar to the test pattern in PHS, where the required revolution speed for keeping a certain constant pump pressure level is measured.

For confidentiality reasons, we are not allowed to use true physical units of the measurements and the results. Therefore, all values are normalized with respect to a nominal value.

The paper is structured as follows: in Section 2, internal gear pumps are briefly explained and the problem is stated. The experimental setup for acquiring test data is introduced in Section 3. Section 4 describes the extraction of the PRTMV as a degradation indicator, and test results are provided in Section 5. Finally, Section 6 gives the conclusions of the work.

\section{Problem Statement}

This section provides a brief introduction of internal gear pumps and a statement about the problem to be solved.

\subsection{Internal Gear Pumps}

In gear pumps, rotating gears create a suction at the intake side by separating from each other. Then the gears carry the fluid to the discharge side, where it is displaced. There are two main types of gear pumps: external and internal gear pumps. In internal gear pumps, one gear rotates inside the other. A short description of both types together with a functional sketch can be found in (Wikipedia, 2021).

System dependent, internal gear pumps show advantages compared to external gear pumps. Some of them are: the centric arrangement of the shaft (easy combination of multiple pumps), the longer tooth meshing due to the use of internal and external gears (improving leakage effects), less flow and filling losses (improving the efficiency of the pump) and better noise behavior than external gear pumps. For more detailed information, like geometrical details, physical models, and many more, see (Murrenhoff, 2005), (Rexroth, n.d.) and (Ivantysyn \& Ivantysynova, 1993).

\subsection{Objective}

The objective of this study is to find an indicator that correlates to the wear of the pump. Since the assessment of the true wear state is not a trivial problem, it is assumed to be roughly correlated to the operating time. Therefore, we are looking for features that are closely related to the operating time of the pump. In a data-driven approach, appropriate features to reach that goal are found. That means, real world measurement data with known operating time are analyzed in order to find features that correlate to the operating time. During the development, many possible indicators were defined, extracted and evaluated. Only the best indicator of the evaluation is presented subsequently in Section 4.

\section{EXPERIMENTAL SETUP}

In this section, the test bed for acquiring test data is briefly explained, and a short overview of the test measurements is 


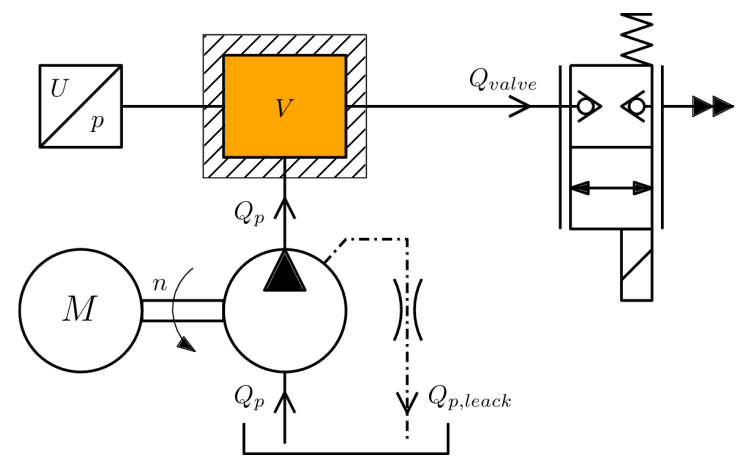

Figure 1. Sketch of the test-bench.

given. Moreover, the basics of the PHS method, that is used for comparison, are presented.

\subsection{Internal Gear Pump Test Bed}

The test bed consists essentially of the motor-pump unit with a rotational speed sensor, a seat type switching valve and a pressure sensor (pressure range $1 . . .413 .7$ bar, analog output range $0.2 \ldots 10.2 \mathrm{~V} \mathrm{DC}$, analog resonance frequency $>10 \mathrm{kHz}$, sampled with 5 kHz @16 Bit), see Fig. 1.

The basic idea is to pressurize the fluid volume $V$ up to a certain pressure level $\hat{p}$ using the pump. The volume depicted in Fig. 1 represents all small volumes, i.e. short bores, dead volumes, and so on, between the pump and the valve. In the case of constant pressure $\hat{p}$ and a closed switching valve, the pumps flow rate $Q_{p}$ compensates its leakage $Q_{p, \text { leak }}$, because the system has no other leaks, writing:

$$
Q_{p}=Q_{p, l e a k}
$$

In that case, the pumps flow rate $Q_{p}$ is proportional to the shaft speed $n$ and we obtain the PHS method.

The starting condition of the PRTMV approach is again constant pressure $\hat{p}$ in the volume. Sudden stopping of the pump, $n=0$, is followed by a rapid reduction of the volumes pressure $p$, which can be measured and processed according to Section 4 . The dynamical change of the pressure can be modeled by:

$$
\frac{d p}{d t}=\frac{E_{0}}{V_{0}} \cdot(\underbrace{Q_{p}}_{=0}-\underbrace{Q_{\text {valve }}}_{=0}-\sum_{i=1}^{N} Q_{i, \text { leak }})
$$

Parameter $E_{0}$ is the fluid's bulk modulus, which is a measure for its compressibility, and $V_{0}$ is the fluid volume. The sum over $Q_{i, l e a k}$, in Eq. (2), indicates that generally, there may be multiple $(N)$ sources of leakage in a system, causing a
Table 1. Tested pumps.

\begin{tabular}{c|c|c}
\hline \hline Pump & Normalized Operating Time & \# measurements \\
\hline \hline$P_{1}$ & 0 & 9 \\
\hline$P_{2}$ & 1 & 9 \\
\hline$P_{3}$ & 0 & 186 \\
\hline$P_{4}$ & 0 & 19 \\
\hline$P_{5}$ & 0 & 247 \\
\hline$P_{6}$ & 0.5442 & 203 \\
\hline$P_{7}$ & 0.5365 & 103 \\
\hline$P_{8}$ & 0.6864 & 92 \\
\hline$P_{9}$ & 0.5306 & 147 \\
\hline$P_{10}$ & 0.4636 & 41 \\
\hline$P_{11}$ & 0.4745 & 98 \\
\hline
\end{tabular}

pressure decay. In the considered case there is only one kind of leakage, the pumps leakage $Q_{p, l e a k}$.

The method can be used as an indicator for the pump wear if the pumps leakage is the main effect causing the pressure drop. Here this is ensured using a seat type switching valve which has construction-related no leakage. Concluding that if the pumps shaft is fixed, the pressure in the volume is only reduced by internal leakage of the pump $Q_{p, l e a k}$, see Fig. 1. In the case of pressure compensated internal gear pumps, the main advantage of this method, compared to the PHS, is that the variable pressure changes the amount of pressure compensation hence having an impact on the pumps leakage. In our assumption, this is the reason why PRTMV shows a better sensitivity than other methods. However, a future task will be to confirm this assumption.

\subsection{Acquired Data}

On the test bed, data from 11 identical pumps with different operating times were acquired. An overview of the tested pumps is provided in Table 1 . Column 1 describes the name of the pump, column 2 represents the pumps operating hours at the beginning of the test, and column 3 represents the number of measurements (i.e. pressure drops) that could be used for evaluation. The operating time is normalized to the range $[0,1]$ with respect to the maximal operating time specified by the manufacturer. A value of 0 represents a new pump, a value of 1 represents a pump that has reached its maximal operating time. From these test data, the PRTMVs are extracted.

\subsection{PHS}

To compare the proposed method to a widely used approach, we also extract the pressure holding speed from the measured data. The PHS represents the revolution speed of the pump that is required to keep a certain pressure $\hat{p}$. It is measured by closing the valves and controlling the pump in a way that the internal pump pressure is kept at a constant level for at least some seconds. Then, the median of the required revolution speed is used as the PHS. As the valves are closed, the pumps 
Table 2. Median normalized feature values of all tested pumps and methods.

\begin{tabular}{c|c|c}
\hline \hline Pump & PRTMV & PHS \\
\hline \hline$P_{1}$ & $4.4605 \cdot 10^{-2}$ & 0.3782 \\
\hline$P_{2}$ & $1.2460 \cdot 10^{-2}$ & 0.8916 \\
\hline$P_{3}$ & $4.4991 \cdot 10^{-2}$ & 0.3696 \\
\hline$P_{4}$ & $4.0655 \cdot 10^{-2}$ & 0.3843 \\
\hline$P_{5}$ & $4.0442 \cdot 10^{-2}$ & 0.3874 \\
\hline$P_{6}$ & $3.0766 \cdot 10^{-2}$ & 0.3908 \\
\hline$P_{7}$ & $2.9647 \cdot 10^{-2}$ & 0.3886 \\
\hline$P_{8}$ & $2.5840 \cdot 10^{-2}$ & 0.4691 \\
\hline$P_{9}$ & $3.0401 \cdot 10^{-2}$ & 0.3982 \\
\hline$P_{10}$ & $2.7946 \cdot 10^{-2}$ & 0.4467 \\
\hline$P_{11}$ & $2.9724 \cdot 10^{-2}$ & 0.4260 \\
\hline
\end{tabular}

flow rate $Q_{p}$ compensates the internal leakage $Q_{p \text {,leak }}$, see also Eq. (1). The flow rate $Q_{p}$ is proportional the the revolution speed $n$. Therefore, rhe more revolution speed is required to keep the pressure constant, the more internal leakage is present. For more details concerning the PHS approach see for instance (Beebe, 2005). We chose PHS to compare it with the proposed PRTMV because it is the most well known and widely used method for pump condition monitoring. Furthermore, there is a clear physical explanation why PHS works to detect a leakage. From our experience, this is also a very important fact for costumers to use PHS as pump monitoring tool. However, the physical interpretability applies also to PRTMV. In (Pichler, Haas, Putz, \& Kastl, 2020) we also compared PHS to another commonly used approach, namely head flow diagrams (HFD). The results showed that PHS and HFD perform quite similar.

\section{Pressure Reduction time Map Volume}

The PRTMV is extracted from the internal pump pressure measurement. It is related to the time needed for the pump pressure dropping from a certain level to another when the pump is stopped and the valves are closed. Since no fluid can flow off through the valves, PRTMV can be seen as representing the internal leakage of the pump. Wear and degradation typically increase the internal leakage, yielding consequently in a decreasing PRTMV.

The extraction of PRTMV is a quite straightforward procedure. The prerequisite for extracting PRTMV is a certain standardized measurement cycle of the system. Once the pump is running on high internal pressure, it is stopped with the valves being closed. The subsequent pressure drop is observed in the raw pressure measurement (Fig. 2 with normalized pressure). The raw data are smoothed using Whittaker smoother (Eilers, 2003) to reduce measurement noise. A zoom into the smoothed pressure drop is shown in Fig. 3.
Since the valves are closed, the pressure drop can only be caused by internal leakage of the pump (see also Section 3 ).

To compute the proposed PRTMV, the time span $t\left(p_{u}, p_{l}\right)$ (pressure reduction time, PRT) that measures the time for the internal pump pressure dropping from a certain upper pressure $p_{u}$ to a certain lower pressure $p_{l}$ is determined (see Fig. 3). Since the measurements are sampled discretely, there will be normally no measurement values exactly at $p_{u}$ and $p_{l}$ and therefore at the according times $t_{u}$ (time when pump pressure equals $p_{u}$ ) and $t_{l}$ (time when pump pressure equals $p_{l}$ ). To get appropriate values for $t_{u}$ and $t_{l}$, the smoothed pressure measurement is linearly interpolated between the adjacent measured pressure values of $p_{u}$ and $p_{l}$. Denoting the adjacent discrete measurement data of the upper pressure value $p_{u}$ as $\bar{p}_{u}$ (upper value) and $\underline{p}_{u}$ (lower value) and the according time values as $\bar{t}_{u}$ and $\underline{t}_{u}$, the linearly interpolated time value $t_{u}$ is given as

$$
t_{u}=\bar{t}_{u}-\frac{\bar{p}_{u}-p_{u}}{\bar{p}_{u}-\underline{p}_{u}} \cdot\left(\bar{t}_{u}-\underline{t}_{u}\right) .
$$

Similarly, the interpolated time value $t_{l}$ is given as

$$
t_{l}=\bar{t}_{l}-\frac{\bar{p}_{l}-p_{l}}{\bar{p}_{l}-\underline{p}_{l}} \cdot\left(\bar{t}_{l}-\underline{t}_{l}\right)
$$

with $\bar{p}_{l}$ and $\underline{p}_{l}$ denoting the adjacent discrete measurement data of the lower pressure value $p_{l}$ and $\bar{t}_{l}$ and $\underline{t}_{l}$ the according times values. The values $p_{u}, p_{l}, t_{u}$ and $t_{l}$ are also indicated in Fig. 3. Finally, $t\left(p_{u}, p_{l}\right)$ is defined as

$$
t\left(p_{u}, p_{l}\right)=t_{l}-t_{u}
$$

This time span is determined for a discrete range of upper and lower pressure values $p_{u} \in\left[P_{l}, P_{u}\right]$ and $p_{l} \in\left[P_{l}, P_{u}\right]$ with $p_{l}<p_{u}$. Here, $P_{u}$ respectively $P_{l}$ denote the maximal respectively minimal pressure to be used for evaluating $t\left(p_{u}, p_{l}\right)$. This procedure results in a pressure reduction time map (PRTM) for one pressure drop of a certain pump. One PRTM in each case of differently worn pumps are shown in Fig. 4 (new pump $P_{1}$ ), Fig. 5 (heavily worn pump $P_{2}$ ), and Fig. 6 (medium worn pump $P_{11}$ ). It can be seen that, especially for high pressure differences $p_{u}-p_{l}$, the new pump $P_{1}$ has a significantly higher pressure reduction time than the worn pumps $P_{2}$ and $P_{11}$.

To quantify this obvious difference, the volume between the $p_{l} p_{u}$-plane and the PRTM is computed as

$$
\operatorname{PRTMV}=\iint_{p_{l}<p_{u}} t\left(p_{u}, p_{l}\right) d p_{l} d p_{u}
$$

The values $p_{l}$ and $p_{u}$ in Eq. (6) indicate the different possible 
Table 3. Relative deviation from the mean of new pumps.

\begin{tabular}{c|r|r}
\hline$\mu \pm \sigma$ for $P_{1}, P_{3}, P_{4}, P_{5}$ & $4.2673 \cdot 10^{-2} \pm 0.2460 \cdot 10^{-2}$ & $0.3798 \pm 0.0078$ \\
\hline \hline Pump & PRTMV & PHS \\
\hline \hline$P_{1}$ & $4.53 \%$ & $-0.44 \%$ \\
\hline$P_{2}$ & $-70.80 \%$ & $134.74 \%$ \\
\hline$P_{3}$ & $5.43 \%$ & $-2.70 \%$ \\
\hline$P_{4}$ & $-4.73 \%$ & $1.16 \%$ \\
\hline$P_{5}$ & $-5.23 \%$ & $1.98 \%$ \\
\hline$P_{6}$ & $-27.90 \%$ & $2.88 \%$ \\
\hline$P_{7}$ & $-30.53 \%$ & $2.29 \%$ \\
\hline$P_{8}$ & $-39.45 \%$ & $23.51 \%$ \\
\hline$P_{9}$ & $-28.76 \%$ & $4.84 \%$ \\
\hline$P_{10}$ & $-34.51 \%$ & $17.60 \%$ \\
\hline$P_{11}$ & $-30.35 \%$ & $12.15 \%$ \\
\hline
\end{tabular}

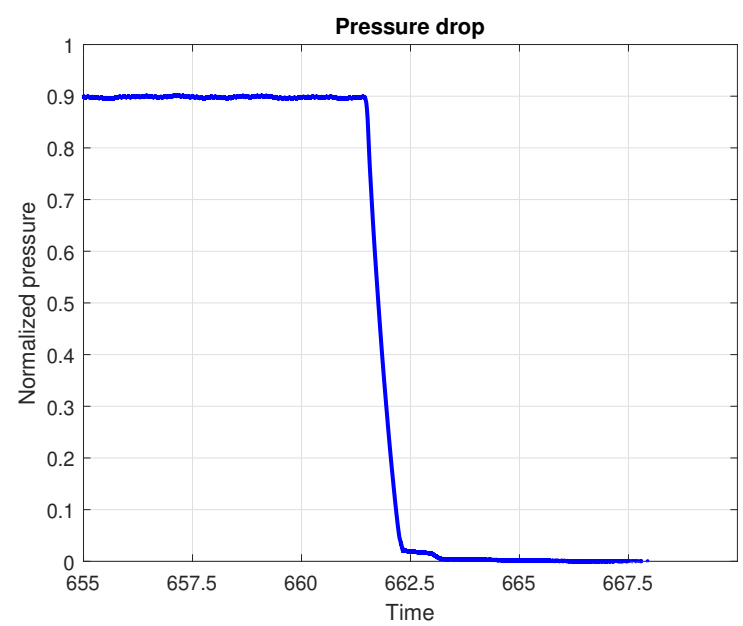

Figure 2. Measured pressure drop of pump $P_{1}$.

pressure values that can be used to estimate $t\left(p_{u}, p_{l}\right)$ according to Eq. (5). Since we measure PRT from $p_{u}$ to $p_{l}, p_{l}$ has to be smaller than $p_{u}$. PRT will get shorter for a degraded pump (Pichler et al., 2020), therefore the integral over all possible pressure drops will be also smaller in the case of degradation. Since we work with discrete measurement values, the integral is approximated by a sum.

As can be seen in the experimental results (Section 5), the PRTMV serves as an indicator that is highly correlated to the operating time of the pump.

\section{RESULTS}

This section provides experimental results for the two approaches to be compared and a short comparison of the results.

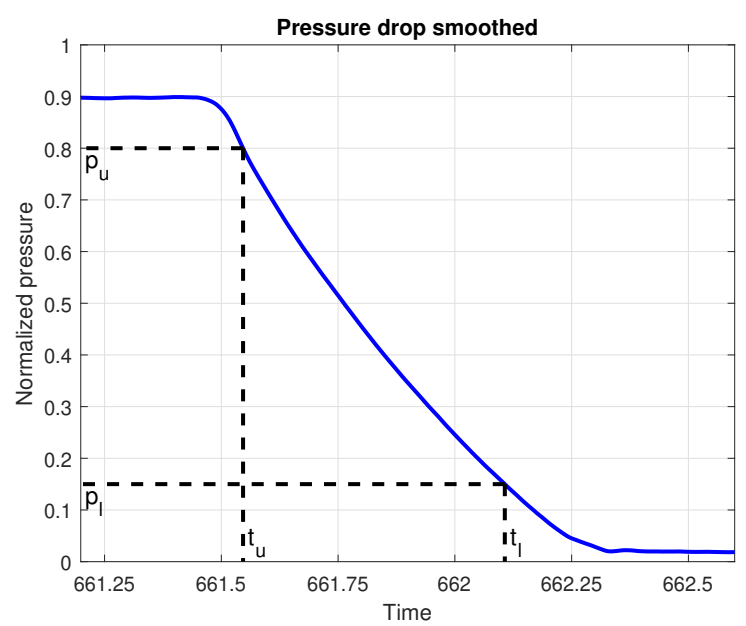

Figure 3. Zoom into smoothed pressure drop from Fig. 2 with indicated values $p_{u}, p_{l}, t_{u}$ and $t_{l}$.

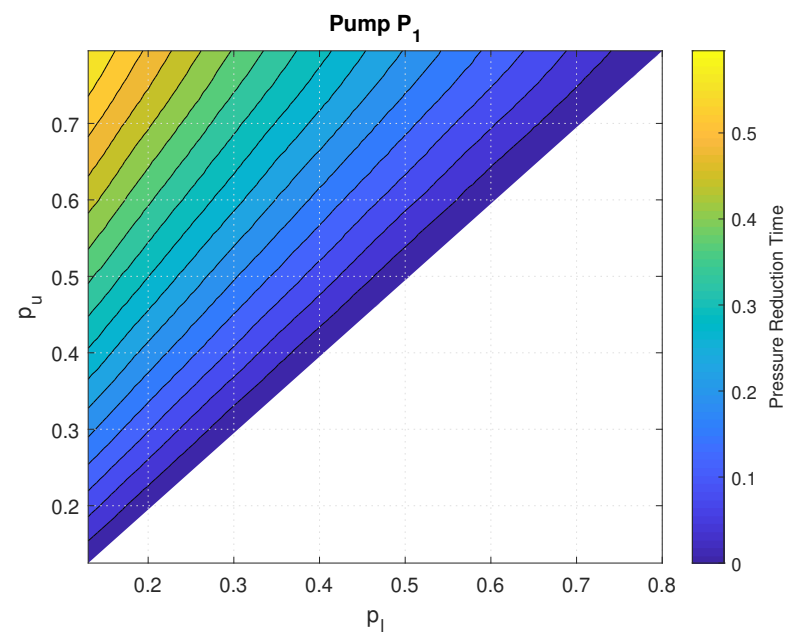

Figure 4. Pressure reduction map for one pressure drop of pump $P_{1}$. 


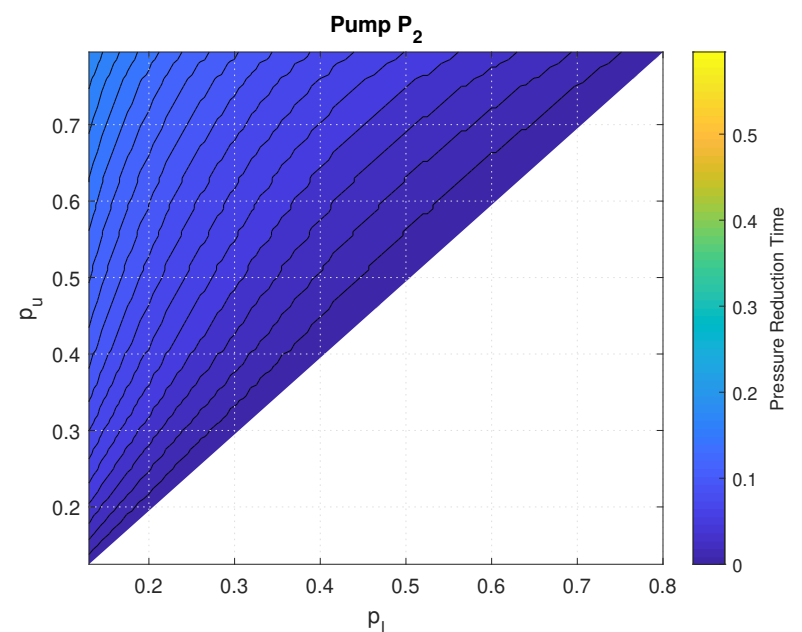

Figure 5. Pressure reduction map for one pressure drop of pump $P_{2}$.

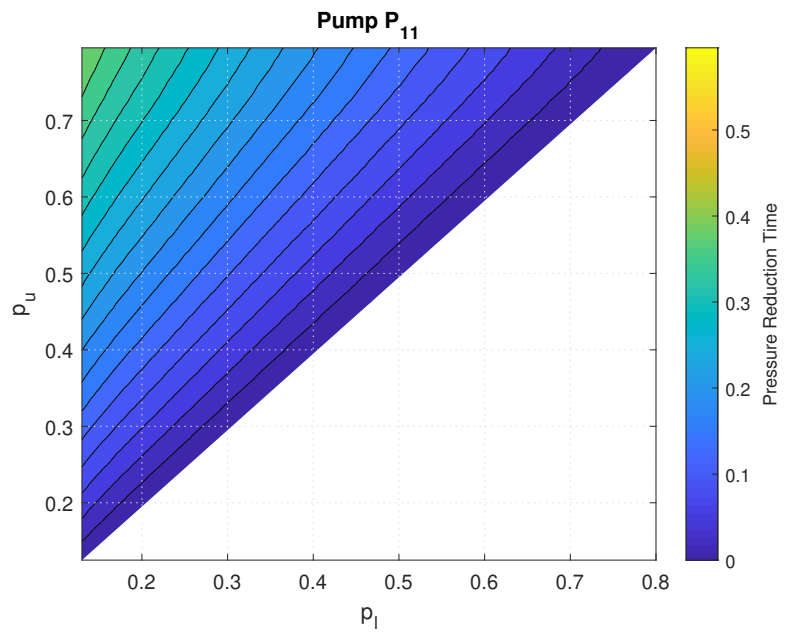

Figure 6. Pressure reduction map for one pressure drop of pump $P_{11}$.

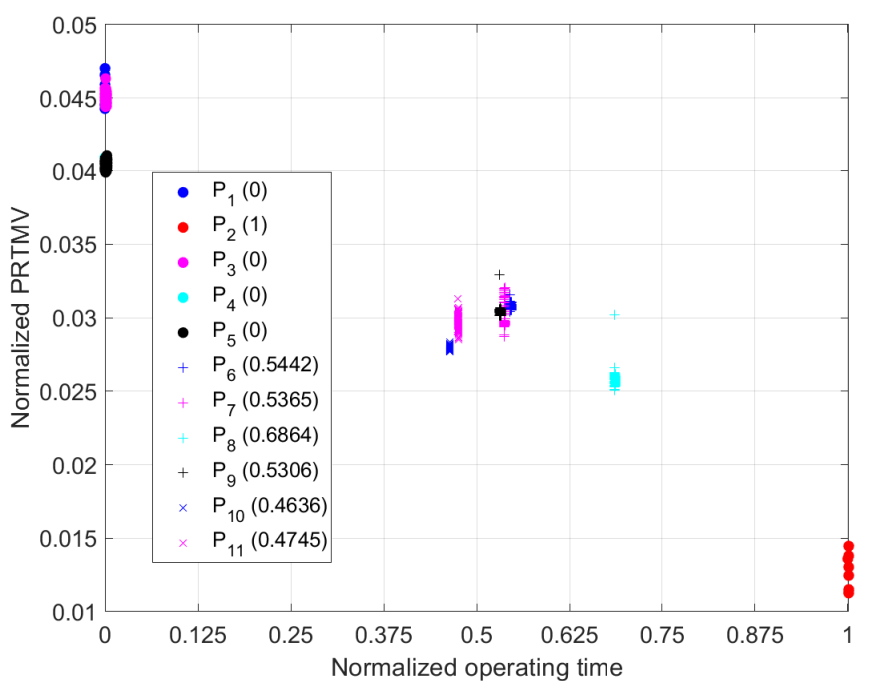

Figure 7. Scatterplot of PRTMV for all tested pumps.

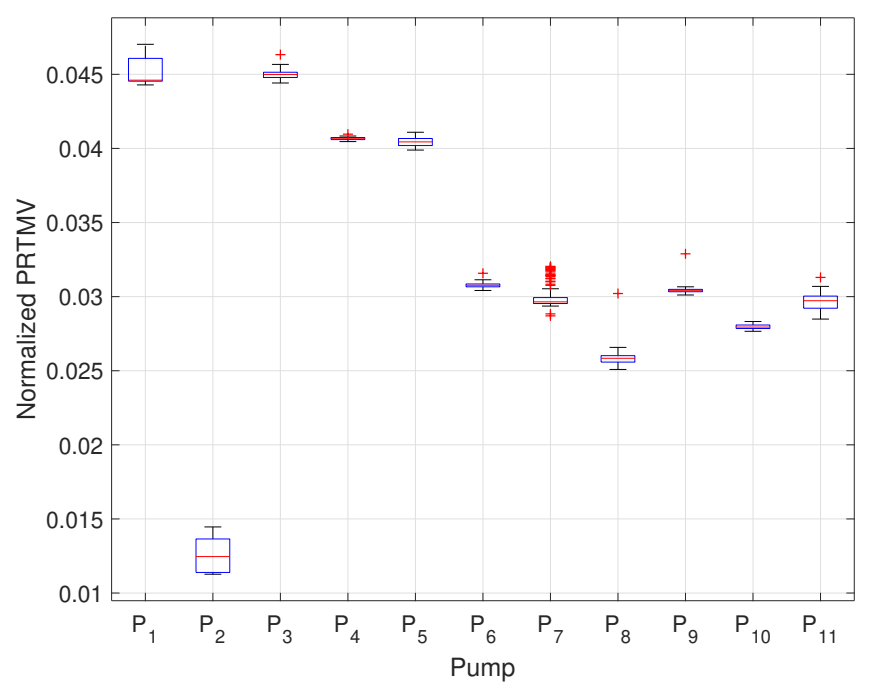

Figure 8. Boxplot of PRTMV for all tested pumps.

\subsection{PRTMV}

Fig. 7 shows a scatterplot of the PRTMV values for all valid measurements of all pumps. It can be seen that the PRTMV (y-axis) is roughly decreasing with increasing operation time (x-axis). Since the data of the new pumps $\left(P_{1}, P_{3}, P_{4}\right.$ and $\left.P_{5}\right)$ are partly overlapping in the scatterplot, a boxplot of the same data is depicted in Fig. 8. Furthermore, the median PRTMV for every pump is listed in the second column of Table 2.

\subsection{PHS}

Scatterplot and boxplot of the feature PHS are depicted in Fig. 9 and Fig. 10 respectively. It can be already seen that PHS is less sensitive than PRTMV for the test cases of medium degradation (normalized operating time between 0.4636 and 0.6864). However, the heavily degraded pump 


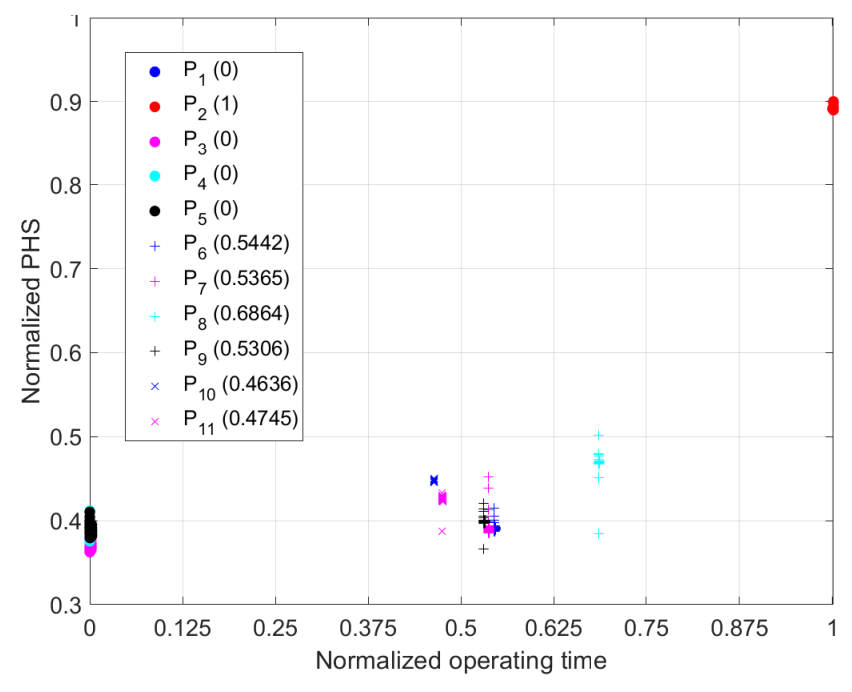

Figure 9. Scatterplot of PHS for all tested pumps.

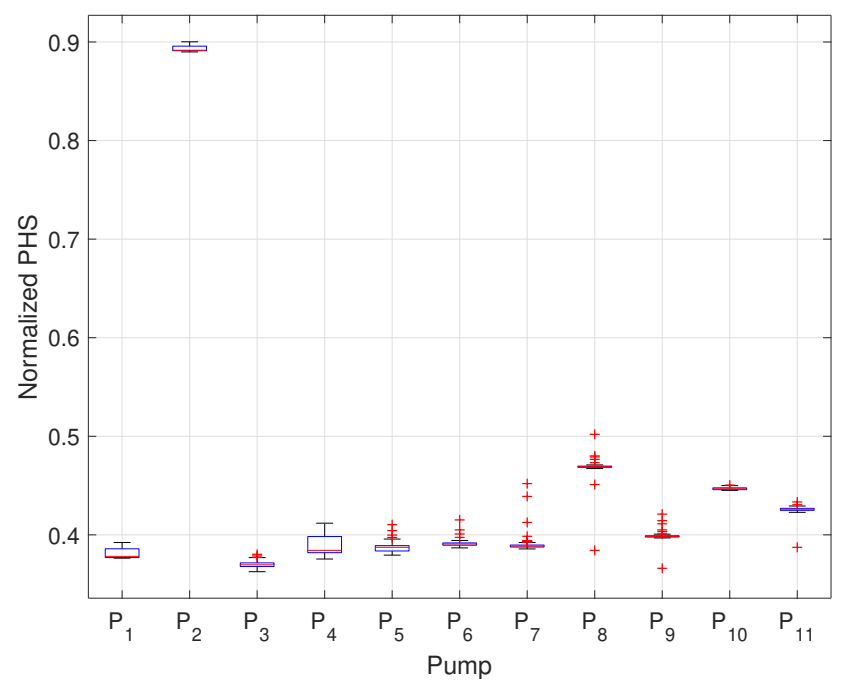

Figure 10. Boxplot of PHS for all tested pumps.

$P_{2}$ (normalized operating time 1) can be clearly identified. Again, the median PHS for every pump is listed in column three of Table 2.

\subsection{Comparison}

To compare the sensitivity of the two approaches, the deviation of PRTMV and PHS from the normal condition is shown in Table 3. For that purpose, the mean indicator values from Table 2 of the new pumps $P_{1}, P_{3}, P_{4}$ and $P_{5}$ (normalized operating time 0 ) are calculated. Table 3 shows these mean values and the according standard deviation in the first row. The relative deviation from that mean value for every pump is listed in the remaining rows. The table shows that, within the pumps with normalized operating time 0 , the proposed PRTMV (absolute deviation between $4.53 \%$ and $5.43 \%$ ) deviates slightly more than the PHS (absolute deviation be- tween $0.44 \%$ and $2.70 \%$ ). Even the minimal deviation of PRTMV is bigger than the maximal deviation of PHS. However, for the medium normalized operation time (between 0.4636 and 0.6864 ) of $P_{6}, P_{7}, P_{8}, P_{9}, P_{10}$ and $P_{11}$, PRTMV is much more sensitive than PHS. That is the main advantage of the proposed approach. PRTMV deviates significantly more from the normal case (between $27.90 \%$ and 39.45\%) than PHS (between $2.29 \%$ and $23.51 \%$ ). This suggests that upcoming wear can be detected earlier and with higher confidence using the PRTMV approach. In the case of heavy degradation (pump $P_{2}$ ), the feature PHS deviates more from the normal condition than PRTMV. The heavy degradation can still be clearly identified by both approaches.

\section{Conclusion}

In this paper, we propose a novel approach to detect wear in internal gear pumps. The proposed PRTMV is a measure related to the time for internal pressure reduction of a pump when the pump engine is stopped and the valves are closed. It was tested with data of real pumps and compared to the well known and commonly used approach PHS. Especially in the range of medium degradation of the pump, the proposed PRTMV is more sensitive than PHS.

However, there are some tasks to be solved in future development of the approach. PRTMV deviates more for normal conditions than PHS. Choosing the pressure range in a more advanced way might improve the deviation. Furthermore, a method for automated wear estimation based on the feature value has to be found. Additionally, a prediction for the maintenance interval based on PRTMV is desirable.

\section{ACKNOWLEDGMENT}

This work has been supported by the COMET-K2 Center of the Linz Center of Mechatronics (LCM) funded by the Austrian federal government and the federal state of Upper Austria.

\section{REFERENCES}

Ahonen, J. (2011). Monitoring of centrifugal pump operation by a frequency converters (Unpublished doctoral dissertation). Lappeenranta University of Technology.

Albraik, A., Althobiani, F., Gu, F., \& Ball, A. (2012). Diagnosis of centrifugal pump faults using vibration methods. Journal of Physics: Conference Series, 364.

Beebe, R. S. (2005). Predictive maintenance of pumps using condition monitoring. Oxford, UK: Elsevier Advanced Technology.

Beebe, R. S. (2008). Experiences in pump condition monitoring by performance analysis. Maintenance \& Asset Management, 23(3), 35-38.

Byington, C. S., Watson, M., Edwards, D., \& Dunkin, B. 
(2003). In-line health monitoring system for hydraulic pumps and motors. In Proceedings of ieee aerospace conference.

Cotogno, M., Cocconcelli, M., \& Rubini, R. (2013). Development of a monitoring system for air bubbles detection in an internal gears pump. In Proceedings of surveillance international conference.

Dutta, N., Umashankar, S., Shankar, V. K. A., Padmanaban, S., Leonowicz, Z., \& Wheeler, P. (2018). Centrifugal pump cavitation detection using machine learning algorithm technique. In Proceedings of ieee international conference on environment and electrical engineering and ieee industrial and commercial power systems europe.

Eilers, P. H. C. (2003). A perfect smoother. Analytical Chemistry, 75(14), 3631-3636.

Harihara, P., \& Parlos, A. G. (2012). Fault diagnosis of centrifugal pumps using motor electrical signals. In D. Papantonis (Ed.), Centrifugal pumps. IntechOpen.

Ivantysyn, J., \& Ivantysynova, M. (1993). Hydrostatische pumpen und motoren. Vogel Fachbuch.

Mendel, E., Wandekokem, E. D., Rauber, T. W., \& Varejão, F. M. (2007). Model-free motor pump fault diagnosis using automatic feature model generation. The Online Journal on Computer Science and Information Technology, 1-3, 71-77.

Murrenhoff, H. (2005). Grundlagen der fluidtechnik, teil 1: Hydraulik. Shaker Verlag.

Pichler, K., Haas, R., Putz, V., \& Kastl, C. (2020). Detecting wear in internal gear pumps by observing the pressure reduction time. In Proceedings of ieee/asme international conference on advanced intelligent mechatronics (aim).

Pietkiewicz, P. (2009). Typical failures of gear pumps - defects classification. Technical Sciences(12), 219-228.

Rexroth. (n.d.). Der hydraulik trainer, band 1 (Tech. Rep. No. ISBN: 3-8023-0619-8). Bosch Group.

Sanchez, W., Carvajal, C., Poalacin, J., \& Salazar, E. (2018). Detection of cavitation in centrifugal pump for vibration analysis. In Proceedings of international conference on control, automation and robotics.

Singh, K., Tjahjowidodo, T., Eriksson, T., \& Rajan, B. (2018). Parameters identification and adaptation for condition monitoring of a reciprocating pump via torque analysis. In Proceedings of ieeelasme international conference on advanced intelligent mechatronics.

Siyuan, L., Linlin, D., \& Wanlu, J. (2018). Study on application of principal component analysis to fault detection in hydraulic pump. In Proceedings of international conference on fluid power and mechatronics.

Sun, H., Yuan, S., \& Luo, Y. (2018). Cyclic spectral analysis of vibration signals for centrifugal pump fault characterization. IEEE Sensors Journal, 18(7), 2925-2933.
Wang, H. Q., \& Chen, P. (2007). Fault diagnosis of centrifugal pump using symptom parameters in frequency domain. Agricultural Engineering International: the CIGR Ejournal, IX.

Wikipedia. (2021). Gear pump. https:// en.wikipedia.org/wiki/Gear_pump. (Accessed: 2021-09-13)

Zouari, R., Sieg-Zieba, S., \& Sidahmed, M. (2004). Fault detection system for centrifugal pumps using neural networks and neuro-fuzzy techniques. In Proceedings of surveillance international conference.

\section{BIOGRAPHIES}

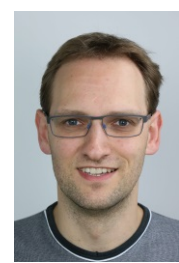

Kurt Pichler was born in Grieskirchen, Austria, in 1981. He received the Dipl-Ing. degree in industrial mathematics from Johannes Kepler University (JKU) Linz, Austria, in 2005, and the Dr. techn. degree in technical sciences from JKU in 2014. He is currently pursuing the MSc degree in artificial intelligence at the JKU. He joined the Area of Sensors \& Communication, Linz Center of Mechatronics $\mathrm{GmbH}$, Linz, in 2007 and works currently as a senior engineer and project leader. His research interests include feature engineering, machine learning, fault diagnosis and artificial intelligence.

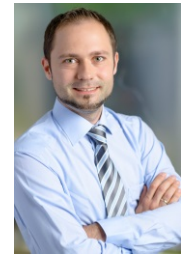

Rainer Haas received the Dipl.-Ing. degree in Mechatronics from the Johannes Kepler University (JKU) in 2007, and the Dr. techn. degree in Mechatronics from JKU in 2013. He joined the area of Drives at Linz Center of Mechatronics, in 2013 and works currently as a senior engineer. Current research interests are hydraulic drives, machine learning, system and software design.

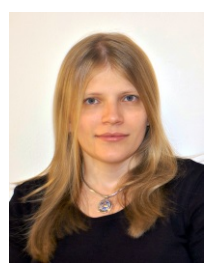

Veronika Putz received the Dipl.-Ing. degree in Electrical Engineering - Audio Engineering from the Technical University of Graz, Austria, in 2004 and the Dr.-techn. degree in Technical Sciences from the Johannes Kepler University of Linz, Austria, in 2010. She joined the Linz Center of Mechatronics in 2012 and currently works as team leader. Her research interests include sensors and measurement technology and digital signal and image processing.

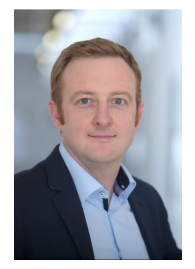

Christian Kastl was born in Linz, Austria, in 1982. He received the B.Sc. degree in mechatronics and microsystems engineering from the University of Applied Sciences Wiener Neustadt, Wiener Neustadt, Austria, in 2011, and the Dipl.Wirtsch.Ing. (FH) degree in industrial engineering from Hamburger Fern-Hochschule, Hamburg, Germany, in 2013. Until 2017, he was the CEO of the company KC-Parts e.U., Linz, which he founded in 2015. He is currently the head of the Department of Sensors and Communication, Linz Center of Mechatronics $\mathrm{GmbH}$, Linz, which he joined in 2011. His research interests comprise distributed computing and symbiotic mechatronics. 